OPEN ACCESS

Edited by:

Giuseppe Marcolin,

University of Padua, Italy

Reviewed by:

Mathew Hill,

Coventry University,

United Kingdom

Guillermo Mendez-Rebolledo,

Santo Tomás University, Chile

*Correspondence:

Nejc Šarabon

nejc.sarabon@fvz.upr.si

Specialty section:

This article was submitted to

Exercise Physiology,

a section of the journa

Frontiers in Physiology

Received: 05 June 2021

Accepted: 26 July 2021

Published: 18 August 2021

Citation:

Kozinc Ž, TrajkovićN, Smajla D and

Šarabon N (2021) The Effect of

Fatigue on Single-Leg Postural Sway

and Its Transient Characteristics in

Healthy Young Adults.

Front. Physiol. 12:720905.

doi: 10.3389/fphys.2021.720905

\section{The Effect of Fatigue on Single-Leg Postural Sway and Its Transient Characteristics in Healthy Young Adults}

\author{
Žiga Kozinc ${ }^{1,2}$, Nebojša Trajković, ${ }^{3}$ Darjan Smajla ${ }^{1,4}$ and Nejc Šarabon ${ }^{1,4,5 *}$ \\ ${ }^{1}$ Faculty of Health Sciences, University of Primorska, Izola, Slovenia, ${ }^{2}$ Andrej Marušič Institute, University of Primorska, Koper, \\ Slovenia, ${ }^{3}$ Faculty of Sport and Physical Education, University of Niš, Niš, Serbia, ${ }^{4}$ Human Health Department, InnoRenew \\ CoE, Izola, Slovenia, ${ }^{5}$ Laboratory for Motor Control and Motor Behavior, S2P, Science to Practice, Ltd., Ljubliana, Slovenia
}

Neuromuscular fatigue is known to impair balance ability, which is reflected in increased postural sway during quiet standing tasks. Recently, quantifying transient characteristics of postural sway has been suggested as an approach to obtain additional information regarding postural control. However, this approach is currently vastly unexplored. The purpose of this study was to investigate the effects of fatigue (induced by a repeated change of direction task) on postural sway and its transient characteristics during single-leg standing, including whole-trial estimates and indexes of transient behavior in young healthy active adults. The study involved 28 physically active students (14 females). Single-leg postural sway was recorded for 30 s before and after a fatiguing protocol, which consisted of a repeated change of direction tasks. We calculated the traditional whole-trial estimates of postural sway [center-of-pressure (CoP) velocity and amplitude in anterior-posterior $(\mathrm{AP})$ and medial-lateral $(\mathrm{ML})$ directions] and corresponding transient behavior indexes, based on three 10-s intervals. Statistically significant sex $\times$ fatigue interaction with medium effect sizes was found for whole-trial CoP velocity in AP $\left(p=0.028 ; \eta^{2}=0.17\right)$ and $\mathrm{ML}$ directions $\left(p=0.019 ; \eta^{2}=0.19\right)$. Post-hoc test showed that both variables substantially decreased in female participants $(p=0.041-0.045 ; d=0.54-0.56)$, but remained similar in males ( $p=0.194-0.294)$. There were small to medium statistically significant main effects of fatigue on transient index for CoP amplitude in both directions $(p=0.042-0.049$; $\left.\eta^{2}=0.02-0.14\right)$. Notably, CoP AP amplitude increased in the first 10-s interval for males (before fatigue: $5.6 \pm 1.3 \mathrm{~mm}$; after fatigue: $6.3 \pm 1.6 \mathrm{~mm}$ ), while the CoP AP amplitude in the third interval remained similar after fatigue (before fatigue: $5.5 \pm 1.4 \mathrm{~mm}$; after fatigue: $5.1 \pm 1.2 \mathrm{~mm})$. In conclusion, the responses to fatigue in terms of postural sway were time interval specific, and there were certain sex-differences in responses to fatigue, which could be related to better ability to adapt balance strategies in females. Moreover, our results demonstrate that the indexes of transient behavior could perhaps detect smaller fatigue-induced changes in postural sway that are seen in whole-trial estimates.

Keywords: body sway, posture, stability, transient behavior, fatiguing 


\section{INTRODUCTION}

Balance and postural control are fundamental abilities underpinning normal human movement function (Pollock et al., 2000; Ivanenko and Gurfinkel, 2018). Assessments of balance are routinely performed in research and clinical practice, studying patients with neuromuscular impairments (Gunn et al., 2015; Shen et al., 2016), children (Geldhof et al., 2006; Alsakhawi and Elshafey, 2019), and older adults (Park, 2018; Kozinc et al., 2020). In athletes, dynamic balance tests have been used to assess injury risk (De Noronha et al., 2006; Plisky et al., 2006); however, the utility of static balance tests (e.g., assessment of postural sway during quiet standing) for athletic populations has not been explored as much. In a literature review, Hrysomallis (2011) reported that static and dynamic balance are related to athletic performance and that balance exercise can contribute to improvements in the performance of movement tasks such as jumping and agility tests. Clearly, the role of balance assessment should not be neglected in athletes, both from injury prevention and performance enhancement aspects. It has been postulated that neuromuscular fatigue, owing to its effects on movement biomechanics, plays an important role in injury risk in sport (Barber-Westin and Noyes, 2017; Benjaminse et al., 2019; Bourne et al., 2019). Therefore, it could be of particular interest to understand how neuromuscular fatigue affects balance.

Static balance is traditionally assessed by measuring postural sway during quiet stance, most typically through center-ofpressure (CoP) movement recordings. The negative effect of neuromuscular fatigue on balance is very well-known. These effects may be driven by various mechanisms, such as altered position sense (Forestier et al., 2002), changes in visual, vestibular, and proprioceptive sensory inputs (Lepers et al., 1997), and reductions in muscle output (Enoka and Stuart, 1992); for review, see Paillard (2012). Increases of postural sway during parallel and single-leg stance have been documented after locally fatiguing lower limb (Lin et al., 2009; Bisson et al., 2011; Zech et al., 2012; Boyas et al., 2013; Barbieri et al., 2019) and after fatiguing the trunk muscles (Pline et al., 2006; Lin et al., 2009; Ghamkhar and Kahlaee, 2019). Similarly, postural sway during single-leg stance is increased after global fatiguing protocols, based on running (Zech et al., 2012; Steib et al., 2013; Beurskens et al., 2016), rowing (Springer and Pincivero, 2009), load lifting (Bannon et al., 2018), and repeated sprinting (Pau et al., 2014). In addition, isolated fatiguing of inspiratory muscles was also reported to result in increased postural sway during parallel stance (Janssens et al., 2010). Since sports training and competitions mostly include full-body movement tasks, it seems reasonable to assume that investigating the effect of global fatigue on balance has greater ecological validity and practical relevance for athletes. In the study performed by Zech et al. (2012), the global fatigue had a significantly larger effect on single-leg postural sway $(+47 \%)$ than local fatigue $(+10 \%)$, whereas similar impairments in dynamic balance (assessed through star excursion balance test) were observed after local and global fatigue in another study (Garcia-Gallart and Encarnacion-Martinez, 2019). Greig and Walker-Johnson (2007) investigated the effect of a 90 -min running protocol on single-leg postural sway in male soccer players and reported that postural sway was not meaningfully affected. On the other hand, an increase in single-leg postural sway was produced by a zig-zag task in male and female athletes from field-based team sports (Lacey and Donne, 2019). Interestingly, aerobic fatigue had a more pronounced effect on single-leg postural sway than anaerobic fatigue in a study conducted on female soccer players (Güler et al., 2020). Recently, Bedo et al. (2020) used a complex fatiguing protocol, consisting of multiple tasks, such as jumping, sidestep cutting, sprinting, and landing. This protocol resulted in a large increase in single-leg postural sway, along with a decrement in maximal strength. Both abilities, however, returned to baseline within $5 \mathrm{~min}$ (Bedo et al., 2020). On the contrary, Baghbani et al. (2016) observed no effect of a complex sevenstation fatigue protocol on dynamic balance (star excursion test in athletes), while the non-athlete control group did perform worse after fatiguing as expected.

The evidence regarding the differences between the sexes in terms of postural sway are somewhat ambiguous, as previous experiments showed either no differences between males and females (Cruz-Gómez et al., 2011; Olchowik et al., 2015) or that females exhibit lower postural sway (Greve et al., 2013; Sell et al., 2018). Caution is needed when examining sex differences, as anthropometric variables may cofound the results (Era et al., 1996; Bryant et al., 2005; Villarrasa-Sapiña et al., 2016; Plandowska et al., 2019). Studies have also suggested that postural sway in males is more sensitive to fatigue (Bannon et al., 2018) and vision elimination (Masui et al., 2005). Therefore, when examining the effects of fatigue on postural sway, sex, and anthropometric variables should be considered as factors in statistical analyses.

Having in mind that balance has been linked to sports performance (Hrysomallis, 2011) and injury risk (Hrysomallis, 2007); the effect of fatigue-induced balance deterioration on injury risk and sports performance is unknown. Recently, a new approach to analyze transient behavior of CoP data has been proposed by Reed et al. (2020). Instead of averaging the CoP data across the trial, this approach separates the data into several time intervals and evaluates the changes in CoP behavior among these intervals. Reed et al. (2020) reported that indexes of the transient behavior of postural sway were independent of the whole-trial estimates (in other words, they were not correlated with the whole-trial estimates), and were sensitive to age and vision elimination. In our subsequent studies, we confirmed the independence of transient indexes and the whole-trial variables, and demonstrated their sensitivity (albeit with small effect sizes) to leg preference (Kozinc and Šarabon, 2021a). Moreover, we found different transient behavior (i.e., quicker reduction of postural sway throughout the trial) in ballet dancers compared to young adults (Kozinc and Šarabon, 2021b). Admittedly, averaging the data is superior in terms of the reliability of the outcomes, however, indexes of transient behavior provide additional information regarding an individual's balance control that are masked (e.g., initial less stable period) by averaging the whole-trial data. This approach could be relevant for injury risk screening in sport. However, we found no differences in terms of the transient behavior of postural sway 
in single-leg stance between athletes with and without ankle sprain history (Kozinc et al., 2021). In this study, we investigated whether indexes of transient behavior of single-leg postural sway could be used to detect deterioration in balance, induced by fatigue. Since fatigue is a major determinant of injury risk in athletes (Benjaminse et al., 2019; Bourne et al., 2019), sensitive and valid approaches to monitor it, are needed. Indexes of transient behavior could be particularly useful if they showed higher sensitivity to fatigue in comparison to whole-trial estimates postural sway. Therefore, the purpose of this study was to investigate the effects of whole-body fatigue on postural sway during single-leg standing, including whole-trial estimates and indexes of transient behavior in young healthy active adults. Based on the large body of literature on the effects of fatigue on postural sway, we considered the single-leg stance as the most appropriate task, being more challenging than bipedal tasks and consequently being expected to be more sensitive to fatigue. We hypothesized that the fatiguing protocol will result in increased whole-trial $\mathrm{CoP}$ amplitude and velocity. The lack of previous studies precluded a formation of hypothesis regarding the effects of fatigue on indexes of transient behavior. The secondary aim of this study was to examine if the effects of fatigue on postural sway are sex-specific.

\section{MATERIALS AND METHODS}

\section{Participants}

Based on the previous studies (Zech et al., 2012; Steib et al., 2013; Pau et al., 2014; Lacey and Donne, 2019; Güler et al., 2020), we expected to observe an increase in CoP velocity and amplitude with an effect size (Cohen's $d$ ) of $\sim 0.75-1.00$. We calculated that 19 participants would be needed to confirm such an effect with $90 \%$ statistical power and alpha level of 0.05 . However, since the fatiguing protocols as well as postural tasks were different in previous studies, we aimed for a higher sample size to detect potential smaller effect. Thus, the study sample comprised of 28 kinesiology students ( 14 females, age: $22.1 \pm 2.1$ years; body mass: $60.5 \pm 5.7 \mathrm{~kg}$; body height: $166.9 \pm 8.4 \mathrm{~cm}$; body mass index: $21.8 \pm 2.4$; and 14 males, age: $26.0 \pm 5.1$ years; body mass: $76.6 \pm 6.7$; body height: $181.5 \pm 4.6 \mathrm{~cm}$; body mass index: $24.2 \pm 2.1$ ). The participants were required to be free from musculoskeletal injuries in the previous 6 months, pain and other medical conditions that could affect postural control. All participants reported being physically active at least three times a week, with different preferred exercises of choice (e.g., resistance training and aerobic exercise). They also completed several study courses that required them to exhibit decent levels of aerobic endurance, strength, flexibility, and balance. For instance, they were required to reach the norms for $2,400 \mathrm{~m}$ run test (males: $<10 \mathrm{~min} 30 \mathrm{~s}$; females: $<11 \mathrm{~min} 30 \mathrm{~s}$ ), hamstring flexibility [reaching to the floor with fingertips (males) or full palms (females) from a standing position, without flexing the knees], and full body strength (lifting 50\% of their body weight three times with an overhead squat exercise technique), and correctly perform several drills or movement skills (swimming techniques, basic elements of soccer, volleyball, basketball and handball, and athletic drills). The procedures of this study were approved by National Medical Ethics Committee (approval number: 0120-690/2017/8) and were conducted in accordance to the Declaration of Helsinki. After the explanation of the experimental procedures, a written informed consent was obtained from all participants.

\section{Study Design and Experimental Tasks}

The study was conducted in a single session, lasting approximately $1 \mathrm{~h}$. Before the measurements, the participants performed a 15-min warm up, consisting of $10 \mathrm{~min}$ of light-intensity running on an indoor track and $5 \mathrm{~min}$ of dynamic stretching exercises. Postural sway was assessed during single-leg quiet stance before and after the fatiguing protocol (see below), using a force plate positioned on a parquet floor. Before the main trials, a standardized $20 \mathrm{~s}$ familiarization trial was performed to introduce the task requirements to the participants. For the main trials, the participants were required to maintain a single-leg stance for $30 \mathrm{~s}$. The knee of the stance leg was in an extended position, but it was not allowed to completely lock (i.e., hyperextend). The hip of the other leg was in a neutral position $\left(0^{\circ}\right)$, whereas the knee was flexed at $90^{\circ}$ and was not allowed to be touching the standing leg. Participants looked at a fixed point at an eye level and $\sim 3 \mathrm{~m}$ away from them. The hands were placed on the hips throughout the trials. A 30-s repetition was performed before and after the fatigue. The participants assumed the single-leg position, and the examiner started the acquisition after $\sim 1 \mathrm{~s}$. After the fatiguing protocol was finished, the postural sway assessments were repeated immediately. We analyzed the postural sway on the preferred leg, which was determined as the leg that the participant would use to kick a ball. It has been shown that postural sway is slightly smaller during standing on a preferred leg compared to the non-preferred leg (Kozinc and Šarabon, 2021a).

\section{Fatiguing Protocol}

The fatiguing protocol consisted of two repeated change-ofdirection (CoD) tasks. Both tasks encompassed a $5+5 \mathrm{~m}$ run with either a 90 or $180^{\circ} \mathrm{CoD}$ maneuvers in between, and were performed in both left and right directions. These procedures are producing reliable outcomes (ICC $=0.84-0.96$; inter-trial variation $<2 \%$ ) in our laboratory (Šarabon et al., 2020). The protocol was performed in a gym (parquet floor). The time to complete each repetition was measured with photocell timing gates (Brower Timing Systems, Draper, UT, United States). The gates were placed at about hip height, with the start line being $0.5 \mathrm{~m}$ behind the first timing gate to avoid early triggering. Before the fatiguing protocol, the participants first completed two familiarization trials for each task (two trials for left and right directions each) at 50 and $75 \%$ of their maximal effort. Then, they performed three repetitions with the maximal effort for each task to establish baseline times. The fatiguing protocol involved performing the CoD tasks in an alternating order (e.g., $90^{\circ}$ - left, $90^{\circ}$ - right, $180^{\circ}$ - left, and $180^{\circ}$ - right), with $5 \mathrm{~s}$ breaks in between. The protocol was stopped when the time to complete the task increased by $20 \%$ or more in 
the $180^{\circ}$ turn task for the dominant direction (the direction for which shorter time was recorded at baseline). Right after the protocol stopped, the participants were asked to report their rate of perceived exertion using a $0-10$ scale as suggested by Foster et al. (2001).

\section{Data Processing and Outcome Measures}

The ground reaction force data were collected (sampling frequency: $1,000 \mathrm{~Hz}$ ) by a force platform (model 9260AA; Kistler, Winterthur, Switzerland) and were low-pass filtered (Butterworth, second order, $10 \mathrm{~Hz}$ ), using the manufacturer's MARS Software (Kistler, Winterthur, Switzerland). The data were then processed in the same software in order to obtain CoP velocity [total, anterior-posterior (AP) and medial-lateral (ML)], CoP amplitude (AP and ML), and CoP frequency. The CoP amplitude was defined as the mean amount of the CoP sway in AP or ML direction, calculated as the common length the COP sway trajectory only in the given direction, divided by the number of changes of movement direction. Firstly, we calculated the traditional whole-trial estimates (i.e., averaged CoP characteristics over the whole $30 \mathrm{~s}$ of the trial). In addition, we also calculated the relative differences between the first and the second (DIF_21) and first and third (DIF_31) 10 -s time intervals within the whole trial. For each interval, that data were locally demeaned. The relative differences between the intervals were expressed as percentages (100\% representing no change; $>100 \%$ indicating an increase in time; and $<100 \%$ indicating a decrease in time).

\section{Statistical Analysis}

Statistical analyses were done in SPSS (version 25.0; SPSS Inc., Chicago, United States). Descriptive statistics were calculated and reported as mean \pm SD. The normality of the data distribution was verified with Shapiro-Wilk tests (all $p \leq 0.065)$. A two-way ANOVA with one between-participant factor (i.e., sex) and one within-participant factor (fatigue) was run to explore the effects of sex, fatigue, as well as sex $\times$ fatigue interaction for $\mathrm{CoP}$ variables and indexes of transient behavior (i.e., the DIF_21 and DIF_21). The effect sizes were expressed as partial eta-squared $\left(\eta^{2}\right)$ and interpreted as small $(<0.13)$, medium $(0.13-0.26)$, and large $(>0.26$; Bakeman, 2005). Bonferroni-corrected $t$-test were used to explore the effect of sex separately before and after fatigue, the effects of fatigue for each sex, and the effects of sex on rating of perceived exertion and differences fatiguing protocol duration between the sexes. The effect sizes for $t$-tests were calculated as Cohen's $d(0.0-0.2$ - trivial; 0.2-0.6moderate; 0.6-1.2 - large; >1.2 - very large; Bernards et al., 2017). We also examined the associations between wholetrial variables and corresponding indexes of transient behavior (i.e., the DIF_21 and DIF_21) before and after the fatigue. Correlations were assessed by Pearson's correlation coefficients and interpreted as negligible $(<0.1)$, weak $(0.1-0.4)$, moderate (0.4-0.7), strong (0.7-0.9), and very strong (>0.9; Schober and Schwarte, 2018). For all analyses, the threshold for statistical significance was set at $p<0.05$.

\section{RESULTS}

There were statistically significant differences between the sexes regarding body height $(t=6.1 ; p<0.001)$, body mass $(t=8.6$; $p<0.001)$, and body mass index $(t=2.9 ; p=0.007)$.

\section{Pre-fatigue Analyses}

In the pre-fatigue state, there was a statistically significant moderate correlation between whole-trial CoP ML velocity and its DIF_21 index $(r=0.52 ; p=0.005)$. This association was also statistically significant when assessed separately for females $(r=0.61 ; p=0.018)$, but not for males $(r=0.43 ; p=0.125)$. The associations between the remaining variables and their corresponding DIF_21 or DIF_31 were not statistically significant, regardless if whole sample or separate gender subgroups were analyzed $(r=0.09-0.33 ; p=0.090$ $0.667)$. Before the fatigue, the difference between the genders was only observed for whole-trial CoP amplitude in AP $(p=0.041$; $d=0.71)$ and $\mathrm{ML}$ direction $(p=0.023 ; d=0.91)$, with the females exhibiting lower values (see Table 1 for descriptive statistics). However, when CoP variables were normalized to body height or body mass, there were no differences between the sexes ( $p=0.158-0.434$ and $0.112-0.496$, respectively). There were no statistically significant correlations between height and CoP variables $(r=0.03-0.31 ; p=0.095-0.893)$. None of the transient behavior indexes was different between sexes (all $p \leq 0.288$ ).

\section{Effects of Fatigue on Whole-Trial Estimates}

After the fatiguing protocol, the average rating of perceived exertion score was $8.7 \pm 0.8$ and was not statistically significantly different $(p=0.376)$ between males $(9 \pm 1)$ and females $(9 \pm 1)$. On average, the males and the females performed $30.8 \pm 6.1$ and $28.8 \pm 6.5$ CoD maneuvers, respectively, with the group difference not being statistically significant $(p=0.411)$. The descriptive statistics for all outcome variables related to postural sway before and after fatigue is available in Table 1. Statistically significant gender $\times$ fatigue interaction with medium effect sizes was found for whole-trial CoP velocity in $\operatorname{AP}\left(p=0.028 ; \eta^{2}=0.17\right)$ and $\mathrm{ML}$ directions $(p=0.019$; $\left.\eta^{2}=0.19\right)$. Both interactions were still present when the outcomes were normalized to body height $\left(p=0.018-0.027 ; \eta^{2}=0.17-0.19\right)$ or body mass $\left(p=0.015-0.019 ; \eta^{2}=0.19-0.21\right)$. Post-hoc test showed that the both variables substantially decreased in female participants ( $p=0.041-0.045 ; d=0.54-0.56)$, but remained similar in males $(p=0.194-0.294)$. Although not reaching statistical significance, there were also notable decreases in $\mathrm{CoP}$ AP and ML amplitude in females $(p=0.056-0.058 ; d=0.61-0.62)$.

\section{Effects of Fatigue on Transient Behavior of Postural Sway}

None of the transient behavior indexes showed statistically significant gender $\times$ fatigue interaction. There were small to medium statistically significant main effects of fatigue for DIF_31 for CoP AP as well as DIF_21 for CoP ML amplitude $\left(p=0.042-0.049 ; \eta^{2}=0.02-0.14\right)$. The fatigue decreased the values of DIF_31 and DIF_21, which suggests that after fatigue, the $\mathrm{CoP}$ amplitude dropped to a larger 
TABLE 1 | Postural sway variables and their transient behavior indexes before and after the fatiguing protocol.

\begin{tabular}{|c|c|c|c|c|c|c|c|c|c|c|c|}
\hline \multirow[t]{3}{*}{ Outcome variable } & \multicolumn{4}{|c|}{ Before fatigue } & \multicolumn{4}{|c|}{ After fatigue } & \multicolumn{3}{|c|}{ Effect size $\left(\eta^{2}\right)$} \\
\hline & \multicolumn{2}{|c|}{ Male } & \multicolumn{2}{|c|}{ Female } & \multicolumn{2}{|c|}{ Male } & \multicolumn{2}{|c|}{ Female } & \multirow[b]{2}{*}{ Gender } & \multirow[b]{2}{*}{ Fatigue } & \multirow[b]{2}{*}{ Interaction } \\
\hline & Mean & $S D$ & Mean & $S D$ & Mean & $S D$ & Mean & $S D$ & & & \\
\hline CoP VEL AP (mm/s) & 27.8 & 3.9 & 28.3 & 6.4 & 29.7 & 5.0 & 24.8 & 5.8 & 0.06 & 0.02 & $0.17^{*}$ \\
\hline CoP VEL ML (mm/s) & 32.2 & 5.4 & 31.2 & 6.3 & 34.5 & 7.3 & 27.8 & 6.0 & 0.12 & 0.01 & $0.19^{*}$ \\
\hline CoP AMP AP (mm) & 5.6 & 1.5 & 4.7 & 0.9 & 5.6 & 1.0 & 4.1 & 1.0 & $0.34^{*}$ & 0.06 & 0.06 \\
\hline CoP AMP ML (mm) & 7.9 & 2.1 & 6.3 & 1.2 & 8.1 & 2.6 & 5.4 & 1.6 & $0.30^{*}$ & 0.03 & 0.07 \\
\hline CoP VEL AP - DIF_21 (\%) & 100.1 & 22.3 & 105.3 & 24.8 & 94.1 & 20.3 & 92.1 & 16.1 & 0.00 & 0.12 & 0.02 \\
\hline CoP VEL AP - DIF_31 (\%) & 93.4 & 14.4 & 92.1 & 16.1 & 86.4 & 17.9 & 88.0 & 21.6 & 0.00 & 0.05 & 0.00 \\
\hline CoP VEL ML - DIF_21 (\%) & 97.9 & 18.7 & 102.8 & 20.4 & 98.8 & 24.7 & 87.4 & 18.5 & 0.01 & 0.10 & 0.12 \\
\hline CoP VEL ML - DIF_31 (\%) & 88.6 & 18.0 & 93.0 & 24.4 & 90.1 & 28.1 & 85.6 & 15.5 & 0.00 & 0.01 & 0.02 \\
\hline CoP AMP AP - DIF_21 (\%) & 107.6 & 38.9 & 109.6 & 27.0 & 96.3 & 33.7 & 91.2 & 18.6 & 0.00 & 0.11 & 0.01 \\
\hline CoP AMP AP - DIF_31 (\%) & 99.6 & 18.5 & 92.3 & 17.0 & 83.8 & 27.9 & 83.2 & 22.9 & 0.02 & $0.14^{*}$ & 0.01 \\
\hline CoP AMP ML - DIF_21 (\%) & 101.7 & 26.3 & 107.2 & 24.7 & 94.5 & 30.5 & 85.9 & 26.1 & 0.00 & $0.14^{*}$ & 0.04 \\
\hline CoP AMP ML - DIF_31 (\%) & 95.2 & 29.4 & 93.7 & 36.5 & 90.6 & 39.5 & 84.9 & 27.8 & 0.01 & 0.02 & 0.00 \\
\hline
\end{tabular}

CoP, centre of pressure; VEL, velocity; AMP, amplitude; AP, anterior-posterior; ML, medial-lateral; DIF_21, relative differences between the first and the second 10-s time intervals within the whole trial; and DIF_31, relative differences between the first and the third 10-s time intervals within the whole trial.

"Denotes statistically significant effect $(p<0.05)$.

extent throughout the 30-s trial. Post-hoc t-tests revealed that DIF_31 for CoP AP amplitude was decreased after fatigue in males $(p=0.044 ; d=0.65)$, but not in females $(p=0.310)$. Looking at the descriptive statistics for interval-specific data for males (provided in Table 2) reveals that the effect of fatigue on in DIF_31 was as a result of an increased CoP AP amplitude in the first 10 -s interval in males (before fatigue: $5.6 \pm 1.3 \mathrm{~mm}$; after fatigue: $6.3 \pm 1.6 \mathrm{~mm}$ ), while the CoP AP amplitude in the third interval was slightly decreased after fatigue (before fatigue: $5.5 \pm 1.4 \mathrm{~mm}$; after fatigue: $5.1 \pm 1.2 \mathrm{~mm}$ ).

In contrast to DIF_31 for CoP AP amplitude, the DIF_21 for CoP ML amplitude decreased after fatigue for females $(p=0.024$; $d=0.81$ ), but not for males $(p=0.533)$. This difference in females was a result in slightly increased $\mathrm{CoP}$ ML amplitude in first interval after fatigue (before fatigue: $6.6 \pm 1.8 \mathrm{~mm}$; after fatigue: $6.9 \pm 1.8 \mathrm{~mm}$ ) and concomitant drop during the second interval (before fatigue: $6.2 \pm 1.5 \mathrm{~mm}$; after fatigue: $5.3 \pm 2.0 \mathrm{~mm}$ ). Although no other statistically significant gender-specific effects were found, all data tended to indicate that after fatigue, males exhibit increased postural sway in the early phases of the trial and relatively unchanged postural sway in later phases of the trial. On the other hand, females decreased the postural sway in later phases of the trial, while their early postural sway was relatively unaffected by fatigue.

After fatigue, the correlation between whole-trial CoP ML velocity and its DIF_21 index $(r=0.59 ; p=0.002)$ was still present and was similar in males only $(r=0.55 ; p=0.043)$ and females only $(r=0.57 ; p=0.034)$. There was also a correlation between whole-trial CoP ML amplitude and its DIF_21 index $(r=0.65 ; p=0.001)$, which was also present both in males $(r=0.76 ; p=0.002)$ and females $(r=0.54 ; p=0.048)$.

\section{DISCUSSION}

The purpose of this study was to explore the effects of wholebody fatigue, induced by repeated CoD task on postural sway in single-leg stance and its transient characteristics. Our hypothesis was rejected, as the fatiguing protocol did not result in an increase of postural sway. On the contrary, postural sway was even reduced in females (the difference for CoP velocity, but not $\mathrm{CoP}$ amplitude being statistically significant), but did not change in males. Some of the indexes of transient postural sway behavior were influenced by fatigue. A closer examination of the data reveals that the changes are in accordance with our hypothesis, as the postural sway was more variable across trial after fatigue (in turn, the indexes of transient behavior were lower), with males being significantly affected in particular during the early phases of the trial. Although we expected larger deteriorations in postural sway, this study nevertheless generated two important findings. Firstly, the response to fatigue was time interval specific, which warrants further investigation of the transient behavior in relation to fatigue. Secondly, there were considerable differences between male and female participants in responses to fatigue, which points out that gender-specific effect of fatigue on postural sway.

Contrary to previous findings, some (positive) correlations were present between whole-trial-estimates and corresponding indexes of transient behavior. This implies that individuals with higher postural sway showed less stabilization (i.e., their sway was reduced less or was even increased throughout the trial). However, given that the associations were moderate, and the fact that four previous studies on larger sample sizes found no associations between whole-trial-estimates and corresponding indexes of transient behavior (Reed et al., 2020; Kozinc and Šarabon, 2021a,b; Kozinc et al., 2021), it is still reasonable to conclude that calculating and analyzing indexes of transient behavior can contribute new information to the traditional whole-trial analysis. Future studies investigating this topic should include correlational analysis to resolve this issue further.

The absence of the main effect of fatigue was a surprising finding, given that studies have reported increases in postural sway following aerobic and anaerobic (Fox et al., 2008; Güler 
TABLE 2 | Interval-specific descriptive statistics.

\begin{tabular}{|c|c|c|c|c|c|c|c|c|}
\hline \multirow[t]{3}{*}{ Outcome variable } & \multicolumn{4}{|c|}{ Before fatigue } & \multicolumn{4}{|c|}{ After fatigue } \\
\hline & \multicolumn{2}{|c|}{ Male } & \multicolumn{2}{|c|}{ Female } & \multicolumn{2}{|c|}{ Male } & \multicolumn{2}{|c|}{ Female } \\
\hline & Mean & $S D$ & Mean & $S D$ & Mean & $S D$ & Mean & $S D$ \\
\hline CoP VEL AP first interval (mm/s) & 28.7 & 4.5 & 28.7 & 6.2 & 32.5 & 8.8 & 26.6 & 5.5 \\
\hline CoP VEL AP second interval (mm/s) & 28.3 & 5.4 & 30.1 & 9.1 & 29.6 & 5.7 & 24.5 & 6.7 \\
\hline CoP VEL AP third interval (mm/s) & 26.5 & 4.2 & 26.2 & 6.2 & 27.0 & 3.6 & 23.3 & 7.1 \\
\hline CoP VEL ML first interval (mm/s) & 34.0 & 5.8 & 31.9 & 5.9 & 36.2 & 6.8 & 30.6 & 5.8 \\
\hline CoP VEL ML second interval (mm/s) & 33.0 & 7.3 & 32.7 & 8.8 & 35.5 & 9.8 & 26.8 & 7.7 \\
\hline CoP VEL ML third interval (mm/s) & 29.7 & 5.9 & 29.0 & 7.7 & 31.9 & 8.6 & 26.0 & 6.2 \\
\hline CoP AMP AP first interval (mm) & 5.6 & 1.3 & 4.8 & 1.0 & 6.3 & 1.6 & 4.5 & 1.0 \\
\hline CoP AMP AP second interval (mm) & 6.0 & 2.2 & 5.1 & 1.4 & 5.7 & 1.4 & 4.1 & 1.1 \\
\hline CoP AMP AP third interval (mm) & 5.5 & 1.4 & 4.3 & 1.0 & 5.1 & 1.2 & 3.7 & 1.3 \\
\hline CoP AMP ML first interval (mm) & 8.2 & 2.3 & 6.6 & 1.8 & 8.7 & 2.1 & 6.2 & 1.5 \\
\hline CoP AMP ML second interval (mm) & 8.2 & 2.9 & 6.9 & 1.8 & 8.3 & 3.7 & 5.3 & 2.0 \\
\hline CoP AMP ML third interval (mm) & 7.5 & 2.2 & 5.7 & 1.7 & 7.6 & 3.2 & 5.1 & 1.9 \\
\hline
\end{tabular}

CoP, centre of pressure; VEL, velocity; AMP, amplitude; AP, anterior-posterior; and ML, medial-lateral.

et al., 2020), local and global (Zech et al., 2012), and sportspecific fatiguing (Greig and Walker-Johnson, 2007; Bedo et al., 2020). The effects of fatigue on postural sway could be related to reductions in muscle force producing capacity (Thorlund et al., 2008; Rampinini et al., 2011), which is observed after both central (Gandevia, 2001) and peripheral fatigue (Fitts, 1994). Other possible underlying causes of increases postural sway include changes in proprioception (Proske, 2019), and specifically for global fatigue, the perturbations introduced due to intensified breathing (Janssens et al., 2010). The most reasonable explanation for the lack of effect of fatigue on postural sway in the present study is that the fatiguing protocol was not sufficiently exhaustive to elicit the expected changes in males. The reduced postural sway in females after fatigue is the most difficult to interpret. The reductions of postural sway did not always imply better balance (Cho et al., 2014). It could be that females changed the postural control strategy. Anyway, while the type of fatiguing protocol is an important limitation of this study, at the same time, our results demonstrate that the indexes of transient behavior could perhaps detect smaller fatigue-induced changes that are masked by wholetrial estimates. Looking only at the whole-trial estimates, one could conclude that our protocol did not affect (males) or reduced (females) the postural sway, while the examination of interval-specific values showed increases sway (males) in the early phase of the trial. It is difficult to speculate what effect would be seen if a more exhausting protocol was used or if the participants were professional athletes. Likely, the effects of fatigue would be even smaller, considering that athletes' balance is less influenced by fatigue compared to general population (Baghbani et al., 2016).

We observed considerable differences regarding the responses in postural sway to fatigue between males and females. Previous studies have suggested that there are no differences in postural sway between the genders in the adult population (Hageman et al., 1995; Cruz-Gómez et al., 2011; Olchowik et al., 2015), or that females exhibit lower postural sway (Greve et al., 2013; Błaszczyk et al., 2014; Sell et al., 2018). Smaller postural sway in females has been often attributed to lower body height (Era et al., 1996; Bryant et al., 2005). However, smaller postural sway in females has been found compared to males after adjusting for height (Frandin et al., 1995), and studies have reported no association between anthropometric variables and postural sway (Ekdahl et al., 1989; Ageberg et al., 2001). While the gender differences in postural sway in the rested state could be at least partially influenced by body height, the causes for different responses to fatigue are likely elsewhere. Masui et al. (2005) reported that the postural sway in older adult males was more influenced by the removal of visual information, compared to older adult females. However, since our fatiguing protocol presumably affected proprioception (leaving vison unperturbed), it would be expected that males would be less affected by fatigue if they placed more importance to visual information. One possible explanation for our results is that females are in general better in sensory integration and sensory reweighing, although the available data on adult populations suggest no gender differences (Faraldo-García et al., 2012). Studies in children suggest that females are better at integrating their sensory inputs, while males treat each sensory input more independently (Smith et al., 2012). It could be that when the balance is challenged (e.g., in fatigued conditions, and/or after the transition to a more challenging posture, such as single-leg stance), the females are better and quicker in adapting their sensory integration in order to stabilize the body. Bannon et al. (2018) observed statistically significant increases in postural sway in males, but not in females, after fatiguing load-lifting task. They concluded that females are able to better adapt their balance strategies to fatiguing conditions than males. Indeed, Adlerton et al. (2003) found increased CoP amplitude and trunk accelerations after local ankle fatiguing in females, whereas CoP velocity was decreased, reflecting a change in the postural control strategy.

Some limitations of the study should be acknowledged. The fatigue protocol was somewhat atypical in comparison to previous studies, which warrant further investigation with different (e.g., local and global, aerobic, and anaerobic) fatiguing 
protocols. The participants in our study were physically active young adults, which means that our results cannot be generalized to other populations such as elite athletes or older adults. In addition, it has to be stressed that our results cannot be extrapolated to the dynamic tasks. Namely, some of the previous studies have observed an effect of fatigue on static balance (i.e., increased postural sway in quiet stance; Zech et al., 2012) without concomitant changes in dynamic balance. Moreover, the contribution of individual sensory systems, as well the sensory integration, is probably dependent on the dynamics and difficulty of the task (Bent et al., 2002, 2005). Finally, the reliability of the interval-specific CoP data and indexes of transient behavior is questionable. It is known that trial duration increases the reliability of $\mathrm{CoP}$ metrics (Carpenter et al., 2001). Since only one trial was conducted for this study in each condition, poor reliability could have affected the results. While we cannot calculate the reliability for this specific sample, we found relatively high coefficients of variation (among three repetitions of the 30-s trial) on a sample of 73 male soccer players (unpublished data). Specifically, the coefficients of variation ranged from 9 to $14 \%$ for whole trial CoP velocity and amplitude variables, and from 12 to $27 \%$ for interval-specific data. Indeed, postural sway assessed through CoP movements is highly variable, and coefficient of variation $>10 \%$ is often observed (Hébert-Losier and Murray, 2020). We used one repetition in our protocol because the fatigue could subside quickly after the completion of the fatiguing protocol (Aboodarda et al., 2019; Güler et al., 2020). Nevertheless, more repetitions should be used prior to the fatiguing protocol to increase the rigor of the results. Before the research on the transient behavior of postural sway is advanced, a methodological study is urgently needed to determine the influence of variable choice, postural task, and trial duration (as well as the duration of individual intervals, used to assess transient behavior) on within- and betweensession reliability.

\section{CONCLUSION}

The purpose of this study was to explore the effects of wholebody fatigue on postural sway in single-leg stance and its transient characteristics. Contrary to previous studies, postural sway did not increase with fatigue; rather it was even reduced in females. Some of the indexes of transient postural sway behavior were influenced by fatigue. Postural sway tended to be more variable across the trial after fatigue. This that the

\section{REFERENCES}

Aboodarda, S. J., Fan, S., Coates, K., and Millet, G. Y. (2019). The short-term recovery of corticomotor responses in elbow flexors. BMC Neurosci. 20:9. doi: 10.1186/s12868-019-0492-x

Adlerton, A. K., Moritz, U., and Moe-Nilssen, R. (2003). Forceplate and accelerometer measures for evaluating the effect of muscle fatigue on postural control during one-legged stance. Physiother. Res. Int. 8, 187-199. doi: 10.1002/ pri.289 indexes of transient behavior could perhaps detect smaller fatigue-induced changes that are masked by whole-trial estimates. Males were particularly affected during the early phases of the trial. Thus, we can conclude that the response to fatigue in terms of postural sway is time interval specific, and that there are certain gender differences in responses to fatigue, which could be related to a better ability to adapt balance strategies in females. Transient characteristics of postural sway represent a potentially useful approach to analyze balance and postural control. Further studies will be needed to assess the utility of this approach for assessing sports performance and injury risk.

\section{DATA AVAILABILITY STATEMENT}

The raw data supporting the conclusions of this article will be made available by the authors, without undue reservation.

\section{ETHICS STATEMENT}

The studies involving human participants were reviewed and approved by Republic of Slovenia National Medical Ethics Committee. The patients/participants provided their written informed consent to participate in this study.

\section{AUTHOR CONTRIBUTIONS}

ŽK, NT, and NŠ conceptualized the idea. NT and DS carried out the measurements. NŠ and DS were overviewing the measurement procedures and administration. NT and $\check{Z} \mathrm{~K}$ analyzed the collected data. ŽK wrote the manuscript. NŠ, NT, and DS finalized the manuscript. All authors contributed to the article and approved the submitted version.

\section{FUNDING}

The study was supported by the Slovenian Research Agency through the project TELASI-PREVENT (L5-1845; body asymmetries as a risk factor in musculoskeletal injury development: studying aetiological mechanisms and designing corrective interventions for primary and tertiary preventive care). The funder played no role in conceptualization of the study, data acquisition, and article writing nor any other phase of the study.

Ageberg, E., Zätterström, R., Fridén, T., and Moritz, U. (2001). Individua factors affecting stabilometry and one-leg hop test in 75 healthy subjects, aged 15-44 years. Scand. J. Med. Sci. Sports 11, 47-53. doi: 10.1034/j.1600-0838.2001.011001047.x

Alsakhawi, R. S., and Elshafey, M. A. (2019). Effect of core stability exercises and treadmill training on balance in children with down syndrome: randomized controlled trial. Adv. Ther. 36, 2364-2373. doi: 10.1007/s12325-019-01024-2

Baghbani, F., Woodhouse, L. J., and Gaeini, A. A. (2016). Dynamic postural control in female athletes and nonathletes after a whole-body fatigue protocol. J. Strength Cond. Res. 30, 1942-1947. doi: 10.1519/JSC.0000000000001275 
Bakeman, R. (2005). Recommended effect size statistics for repeated measures designs. Behav. Res. Methods 37, 379-384. doi: 10.3758/BF03192707

Bannon, H. M., Hakansson, N. A., Jakobsen, M. D., Sundstrup, E., and Jorgensen, M. J. (2018). The effects of a fatiguing lifting task on postural sway among males and females. Hum. Mov. Sci. 59, 193-200. doi: 10.1016/j. humov.2018.03.008

Barber-Westin, S. D., and Noyes, F. R. (2017). Effect of fatigue protocols on lower limb neuromuscular function and implications for anterior cruciate ligament injury prevention training: a systematic review. Am. J. Sports Med. 45, 3388-3396. doi: 10.1177/0363546517693846

Barbieri, F. A., Penedo, T., Simieli, L., Barbieri, R. A., Zagatto, A. M., Van Diëen, J. H., et al. (2019). Effects of ankle muscle fatigue and visual behavior on postural sway in young adults. Front. Physiol. 10:643. doi: 10.3389/fphys.2019.00643

Bedo, B. L. S., Pereira, D. R., Moraes, R., Kalva-Filho, C. A., Will-de-Lemos, T., and Santiago, P. R. P. (2020). The rapid recovery of vertical force propulsion production and postural sway after a specific fatigue protocol in female handball athletes. Gait Posture 77, 52-58. doi: 10.1016/j.gaitpost.2020.01.017

Benjaminse, A., Webster, K. E., Kimp, A., Meijer, M., and Gokeler, A. (2019). Revised approach to the role of fatigue in anterior cruciate ligament injury prevention: a systematic review with meta-analyses. Sports Med. 49, 565-586. doi: 10.1007/s40279-019-01052-6

Bent, L. R., McFadyen, B. J., and Inglis, T. J. (2002). Visual-vestibular interactions in postural control during the execution of a dynamic task. Exp. Brain Res. 146, 490-500. doi: 10.1007/s00221-002-1204-8

Bent, L. R., McFadyen, B. J., and Inglis, J. T. (2005). Vestibular contributions during human locomotor tasks. Exerc. Sport Sci. Rev. 33, 107-113. doi: 10.1097/00003677-200507000-00002

Bernards, J., Sato, K., Haff, G., and Bazyler, C. (2017). Current research and statistical practices in sport science and a need for change. Sports 5:87. doi: $10.3390 /$ sports5040087

Beurskens, R., Haeger, M., Kliegl, R., Roecker, K., and Granacher, U. (2016). Postural control in dual-task situations: does whole-body fatigue matter? PLoS One 11:e0147392. doi: 10.1371/journal.pone.0147392

Bisson, E. J., McEwen, D., Lajoie, Y., and Bilodeau, M. (2011). Effects of ankle and hip muscle fatigue on postural sway and attentional demands during unipedal stance. Gait Posture 33, 83-87. doi: 10.1016/j.gaitpost.2010.10.001

Błaszczyk, J. W., Beck, M., and Sadowska, D. (2014). Assessment of postural stability in young healthy subjects based on directional features of posturographic data: vision and gender effects. Acta Neurobiol. Exp. 74, 433-442.

Bourne, M. N., Webster, K. E., and Hewett, T. E. (2019). Is fatigue a risk factor for anterior cruciate ligament rupture? Sports Med. 49, 1629-1635. doi: 10.1007/s40279-019-01134-5

Boyas, S., Hajj, M., and Bilodeau, M. (2013). Influence of ankle plantarflexor fatigue on postural sway, lower limb articular angles, and postural strategies during unipedal quiet standing. Gait Posture 37, 547-551. doi: 10.1016/j. gaitpost.2012.09.014

Bryant, E. C., Trew, M. E., Bruce, A. M., Kuisma, R. M. E., and Smith, A. W. (2005). Gender differences in balance performance at the time of retirement. Clin. Biomech. 20, 330-335. doi: 10.1016/j.clinbiomech.2004.11.006

Carpenter, M. G., Frank, J. S., Winter, D. A., and Peysar, G. W. (2001). Sampling duration effects on centre of pressure summary measures. Gait Posture 13, 35-40. doi: 10.1016/S0966-6362(00)00093-X

Cho, K., Lee, K., Lee, B., Lee, H., and Lee, W. (2014). Relationship between postural sway and dynamic balance in stroke patients. J. Phys. Ther. Sci. 26, 1989-1992. doi: 10.1589/jpts.26.1989

Cruz-Gómez, N. S., Plascencia, G., Villanueva-Padrón, L. A., and Jáuregui-Renaud, K. (2011). Influence of obesity and gender on the postural stability during upright stance. Obes. Facts 4, 212-217. doi: 10.1159/000329408

De Noronha, M., Refshauge, K. M., Herbert, R. D., and Kilbreath, S. L. (2006). Do voluntary strength, proprioception, range of motion, or postural sway predict occurrence of lateral ankle sprain? Br. J. Sports Med. 40, 824-828. doi: $10.1136 /$ bjsm.2006.029645

Ekdahl, C., Jarnlo, G. B., and Andersson, S. I. (1989). Standing balance in healthy subjects. Evaluation of a quantitative test battery on a force platform. Scand. J. Rehabil. Med. 21, 187-195.

Enoka, R. M., and Stuart, D. G. (1992). Neurobiology of muscle fatigue. J. Appl. Physiol. 72, 1631-1648. doi: 10.1152/jappl.1992.72.5.1631
Era, P., Schroll, M., Ytting, H., Gause-Nilsson, I., Heikkinen, E., and Steen, B. (1996). Postural balance and its sensory-motor correlates in 75-year-old men and women: a cross-national comparative study. J. Gerontol. A Biol. Sci. Med. Sci. 51, M53-M63. doi: 10.1093/gerona/51a.2.m53

Faraldo-García, A., Santos-Pérez, S., Crujeiras-Casais, R., Labella-Caballero, T., and Soto-Varela, A. (2012). Influence of age and gender in the sensory analysis of balance control. Eur. Arch. Otorhinolaryngol. 269, 673-677. doi: 10.1007/s00405-011-1707-7

Fitts, R. H. (1994). Cellular mechanisms of muscle fatigue. Physiol. Rev. 74, 49-94. doi: 10.1152/physrev.1994.74.1.49

Forestier, N., Teasdale, N., and Nougier, V. (2002). Alteration of the position sense at the ankle induced by muscular fatigue in humans. Med. Sci. Sports Exerc. 34, 117-122. doi: 10.1097/00005768-200201000-00018

Foster, C., Florhaug, J. A., Franklin, J., Gottschall, L., Hrovatin, L. A., Parker, S., et al. (2001). A new approach to monitoring exercise training. J. Strength Cond. Res. 15, 109-115. doi: 10.1519/00124278-200102000-00019

Fox, Z. G., Mihalik, J. P., Blackburn, J. T., Battaglini, C. L., and Guskiewicz, K. M. (2008). Return of postural control to baseline after anaerobic and aerobic exercise protocols. J. Athl. Train. 43, 456-463. doi: 10.4085/1062-6050-43.5.456

Frandin, K., Sonn, U., Svantesson, U., and Grimby, G. (1995). Functional balance test in 76-year-olds in relation to performance, activities of daily living and platform tests. Scand. J. Rehabil. Med. 27, 231-241. doi: 10.1080/16501977231241 Gandevia, S. C. (2001). Spinal and supraspinal factors in human muscle fatigue. Physiol. Rev. 81, 1725-1789. doi: 10.1152/physrev.2001.81.4.1725

Garcia-Gallart, A., and Encarnacion-Martinez, A. (2019). Peripheral and central fatigue cause similar decreases on dynamic postural stability in male recreational runners. Eur. J. Hum. Mov. 42, 42-58.

Geldhof, E., Cardon, G., De Bourdeaudhuij, I., Danneels, L., Coorevits, P., Vanderstraeten, G., et al. (2006). Static and dynamic standing balance: testretest reliability and reference values in 9 to 10 year old children. Eur. J. Pediatr. 165, 779-786. doi: 10.1007/s00431-006-0173-5

Ghamkhar, L., and Kahlaee, A. H. (2019). The effect of trunk muscle fatigue on postural control of upright stance: a systematic review. Gait Posture 72, 167-174. doi: 10.1016/j.gaitpost.2019.06.010

Greig, M., and Walker-Johnson, C. (2007). The influence of soccer-specific fatigue on functional stability. Phys. Ther. Sport 8, 185-190. doi: 10.1016/j. ptsp.2007.03.001

Greve, J. M. D. A., Cuğ, M., Dülgeroğlu, D., Brech, G. C., and Alonso, A. C. (2013). Relationship between anthropometric factors, gender, and balance under unstable conditions in young adults. Biomed. Res. Int. 2013:850424. doi: $10.1155 / 2013 / 850424$

Güler, Ö., Aras, D., Akça, F., Bianco, A., Lavanco, G., Paoli, A., et al. (2020). Effects of aerobic and anaerobic fatigue exercises on postural control and recovery time in female soccer players. Int. J. Environ. Res. Public Health 17:6273. doi: 10.3390/ijerph 17176273

Gunn, H., Markevics, S., Haas, B., Marsden, J., and Freeman, J. (2015). Systematic review: the effectiveness of interventions to reduce falls and improve balance in adults with multiple sclerosis. Arch. Phys. Med. Rehabil. 96, 1898-1912. doi: 10.1016/j.apmr.2015.05.018

Hageman, P. A., Leibowitz, J. M., and Blanke, D. (1995). Age and gender effects on postural control measures. Arch. Phys. Med. Rehabil. 76, 961-965. doi: 10.1016/S0003-9993(95)80075-1

Hébert-Losier, K., and Murray, L. (2020). Reliability of centre of pressure, plantar pressure, and plantar-flexion isometric strength measures: a systematic review. Gait Posture 75, 46-62. doi: 10.1016/j.gaitpost.2019.09.027

Hrysomallis, C. (2007). Relationship between balance ability, training and sports injury risk. Sports Med. 37, 547-556. doi: 10.2165/00007256-200737060-00007

Hrysomallis, C. (2011). Balance ability and athletic performance. Sports Med. 41, 221-232. doi: 10.2165/11538560-000000000-00000

Ivanenko, Y., and Gurfinkel, V. S. (2018). Human postural control. Front. Neurosci. 12:171. doi: 10.3389/fnins.2018.00171

Janssens, L., Brumagne, S., Polspoel, K., Troosters, T., and McConnell, A. (2010). The effect of inspiratory muscles fatigue on postural control in people with and without recurrent low back pain. Spine 35, 1088-1094. doi: 10.1097/ BRS.0b013e3181bee 5c3

Kozinc, Ž., Löfler, S., Hofer, C., Carraro, U., and Šarabon, N. (2020). Diagnostic balance tests for assessing risk of falls and distinguishing older adult fallers and non-fallers: a systematic review with meta-analysis. Diagnostics 10:667. doi: 10.3390/diagnostics10090667 
Kozinc, Ž., and Šarabon, N. (2021a). The effects of leg preference on transient characteristics of body sway during single-leg stance: a cross-sectional study. Front. Hum. Neurosci. 14:617222. doi: 10.3389/fnhum.2020.617222

Kozinc, Ž., and Šarabon, N. (2021b). Transient body sway characteristics during single-leg quiet stance in ballet dancers and young adults. J. Biomech. 115:110195. doi: 10.1016/j.jbiomech.2020.110195

Kozinc, Ž., Trajković, N., and Šarabon, N. (2021). Transient characteristics of body sway during single-leg stance in athletes with a history of ankle sprain. Gait Posture 86, 205-210. doi: 10.1016/j.gaitpost.2021.03.022

Lacey, M., and Donne, B. (2019). Does fatigue impact static and dynamic balance variables in athletes with a previous ankle injury? Int. J. Exerc. Sci. 12, 1121-1137.

Lepers, R., Bigard, A. X., Diard, J. P., Gouteyron, J. F., and Guezennec, C. Y. (1997). Posture control after prolonged exercise. Eur. J. Appl. Physiol. Occup. Physiol. 76, 55-61. doi: 10.1007/s004210050212

Lin, D., Nussbaum, M. A., Seol, H., Singh, N. B., Madigan, M. L., and Wojcik, L. A. (2009). Acute effects of localized muscle fatigue on postural control and patterns of recovery during upright stance: influence of fatigue location and age. Eur. J. Appl. Physiol. 106, 425-434. doi: 10.1007/s00421-009-1026-5

Masui, T., Hasegawa, Y., Matsuyama, Y., Sakano, S., Kawasaki, M., and Suzuki, S. (2005). Gender differences in platform measures of balance in rural communitydwelling elders. Arch. Gerontol. Geriatr. 41, 201-209. doi: 10.1016/j. archger.2005.02.003

Olchowik, G., Tomaszewski, M., Olejarz, P., Warchoł, J., Różańska-Boczula, M., and Maciejewski, R. (2015). The human balance system and gender. Acta Bioeng. Biomech. 17, 69-74. doi: 10.5277/ABB-00002-2014-05

Paillard, T. (2012). Effects of general and local fatigue on postural control: a review. Neurosci. Biobehav. Rev. 36, 162-176. doi: 10.1016/j.neubiorev.2011.05.009

Park, S. H. (2018). Tools for assessing fall risk in the elderly: a systematic review and meta-analysis. Aging Clin. Exp. Res. 30, 1-16. doi: 10.1007/ s40520-017-0749-0

Pau, M., Ibba, G., and Attene, G. (2014). Fatigue-induced balance impairment in young soccer players. J. Athl. Train. 49, 454-461. doi: 10.4085/1062-6050-49.2.12

Plandowska, M., Lichota, M., and Górniak, K. (2019). Postural stability of 5-year-old girls and boys with different body heights. PLoS One 14:e0227119. doi: 10.1371/journal.pone.0227119

Pline, K. M., Madigan, M. L., and Nussbaum, M. A. (2006). Influence of fatigue time and level on increases in postural sway. Ergonomics 49, 1639-1648. doi: 10.1080/00140130600901678

Plisky, P. J., Rauh, M. J., Kaminski, T. W., and Underwood, F. B. (2006). Star excursion balance test as a predictor of lower extremity injury in high school basketball players. J. Orthop. Sports Phys. Ther. 36, 911-919. doi: 10.2519/jospt.2006.2244

Pollock, A. S., Durward, B. R., Rowe, P. J., and Paul, J. P. (2000). What is balance? Clin. Rehabil. 14, 402-406. doi: 10.1191/0269215500cr342oa

Proske, U. (2019). Exercise, fatigue and proprioception: a retrospective. Exp. Brain Res. 237, 2447-2459. doi: 10.1007/s00221-019-05634-8

Rampinini, E., Bosio, A., Ferraresi, I., Petruolo, A., Morelli, A., and Sassi, A. (2011). Match-related fatigue in soccer players. Med. Sci. Sports Exerc. 43, 2161-2170. doi: 10.1249/MSS.0b013e31821e9c5c

Reed, C. A., Chaudhari, A. M. W., Worthen-Chaudhari, L. C., Bigelow, K. E., and Monfort, S. M. (2020). A new perspective on transient characteristics of quiet stance postural control. PLoS One 15:e0237246. doi: 10.1371/journal. pone. 0237246
Šarabon, N., Smajla, D., Maffiuletti, N. A., and Bishop, C. (2020). Strength, jumping and change of direction speed asymmetries in soccer, basketball and tennis players. Symmetry 12:1664. doi: 10.3390/sym12101664

Schober, P., and Schwarte, L. A. (2018). Correlation coefficients: appropriate use and interpretation. Anesth. Analg. 126, 1763-1768. doi: 10.1213/ ANE.0000000000002864

Sell, T. C., Lovalekar, M. T., Nagai, T., Wirt, M. D., Abt, J. P., and Lephart, S. M. (2018). Gender differences in static and dynamic postural stability of soldiers in the Army's 101st airborne division (air assault). J. Sport Rehabil. 27, 126-131. doi: 10.1123/jsr.2016-0131

Shen, X., Wong-Yu, I. S. K., and Mak, M. K. Y. (2016). Effects of exercise on falls, balance, and gait ability in Parkinson's disease. Neurorehabil. Neural Repair 30, 512-527. doi: 10.1177/1545968315613447

Smith, A., Ulmer, F., and Wong, D. (2012). Gender differences in postural stability among children. J. Hum. Kinet. 33, 25-32. doi: 10.2478/ v10078-012-0041-5

Springer, B. K., and Pincivero, D. M. (2009). The effects of localized muscle and whole-body fatigue on single-leg balance between healthy men and women. Gait Posture 30, 50-54. doi: 10.1016/j.gaitpost.2009.02.014

Steib, S., Zech, A., Hentschke, C., and Pfeifer, K. (2013). Fatigue-induced alterations of static and dynamic postural control in athletes with a history of ankle sprain. J. Athl. Train. 48, 203-208. doi: 10.4085/1062-6050-48.1.08

Thorlund, J. B., Michalsik, L. B., Madsen, K., and Aagaard, P. (2008). Acute fatigue-induced changes in muscle mechanical properties and neuromuscular activity in elite handball players following a handball match. Scand. J. Med. Sci. Sports 18, 462-472. doi: 10.1111/j.1600-0838.2007.00710.x

Villarrasa-Sapiña, I., García-Massó, X., Serra-Añó, P., Garcia-Lucerga, C., Gonzalez, L. M., and Lurbe, E. (2016). Differences in intermittent postural control between normal-weight and obese children. Gait Posture 49, 1-6. doi: $10.1016 /$ j.gaitpost.2016.06.012

Zech, A., Steib, S., Hentschke, C., Eckhardt, H., and Pfeifer, K. (2012). Effects of localized and general fatigue on static and dynamic postural control in male team handball athletes. J. Strength Cond. Res. 26, 1162-1168. doi: 10.1519/JSC.0b013e31822dfbbb

Conflict of Interest: NŠ was employed by company S2P, Science to Practice, Ltd.

The remaining authors declare that the research was conducted in the absence of any commercial or financial relationships that could be construed as a potential conflict of interest.

Publisher's Note: All claims expressed in this article are solely those of the authors and do not necessarily represent those of their affiliated organizations, or those of the publisher, the editors and the reviewers. Any product that may be evaluated in this article, or claim that may be made by its manufacturer, is not guaranteed or endorsed by the publisher.

Copyright (๑ 2021 Kozinc, Trajković, Smajla and Šarabon. This is an open-access article distributed under the terms of the Creative Commons Attribution License (CC BY). The use, distribution or reproduction in other forums is permitted, provided the original author(s) and the copyright owner(s) are credited and that the original publication in this journal is cited, in accordance with accepted academic practice. No use, distribution or reproduction is permitted which does not comply with these terms. 